\title{
Curves of constant diameter and inscribed polygons
}

\author{
Mathieu Baillif
}

M. Baillif received his Ph.D. from the University of Geneva in 2002. He now lives in Argentina and does mathematics on his own.

Let $\Gamma$ be a simple closed curve in the Euclidean plane. Say that a polygon $S$ is inscribed in $\Gamma$ at $x$ if all the vertices of $S$ lie on $\Gamma$ and one is $x$. A line segment is here considered as a 2-gon. We say that $\Gamma$ has property $C_{n}(D)$ (for some $n \geq 2, D>0$ ) if

$\forall x \in \Gamma$ there is a unique regular $n$-gon with edges of length $D$ inscribed in $\Gamma$ at $x$.

$\left(C_{n}(D)\right)$

Notice that $C_{2}(D)$ is equivalent to the following since $\Gamma$ is simple and closed $(\|\cdot\|$ is the Euclidean norm):

$$
\begin{aligned}
& \forall x \in \Gamma \quad \exists ! y(x) \in \Gamma \text { with }\|x-y(x)\|=D, \text { and } \\
& \text { if } z \neq y(x), \quad\|x-z\|<D .
\end{aligned}
$$

If one drops the unicity assumption, $C(D)$ is the property of having constant diameter, which is in fact equivalent (for closed curves in the plane) to having constant width or constant breadth (for the definitions and the proof of the equivalence, see [8, chap. 25]). It is a surprise to many (it was to me!) that curves of constant diameter different from the

In der vorliegenden Arbeit sucht der Autor nach einfach geschlossenen Kurven $\Gamma$ in der Euklidischen Ebene, welche die folgende Eigenschaft $C_{n}(D)$ besitzen: Jeder Punkt $x \in \Gamma$ ist Eckpunkt eines der Kurve $\Gamma$ einbeschriebenen regulären $n$-Ecks der Seitenlänge $D$. Im Fall $n=2$ ist dieses Problem im wesentlichen äquivalent mit der Suche nach Kurven konstanter Breite. Diese Problematik wurde bereits von vielen bedeutenden Mathematikern untersucht, z.B. von L. Euler, A. Hurwitz oder H. Minkowski. Mit Hilfe elementarer Methoden zeigt der Autor in dieser Arbeit, dass es $C^{\infty}$-glatte Kurven gibt, welche die Eigenschaft $C_{n}(D)$ besitzen und nicht Kreise sind. Darüber hinaus wird gezeigt, dass der Kreis die einzige zweifach differenzierbare Kurve ist, die sowohl $C_{4}(1 / \sqrt{2})$ als auch $C_{2}(1)$ erfüllt. 
circle do exist. The simplest examples (attributed to Reuleaux [9], but implicit in a much earlier paper by Euler [3]) are pictured in Fig. 1. They are built with circle arcs whose centers are marked with a black dot. Notice that these curves are not $\mathcal{C}^{2}$.
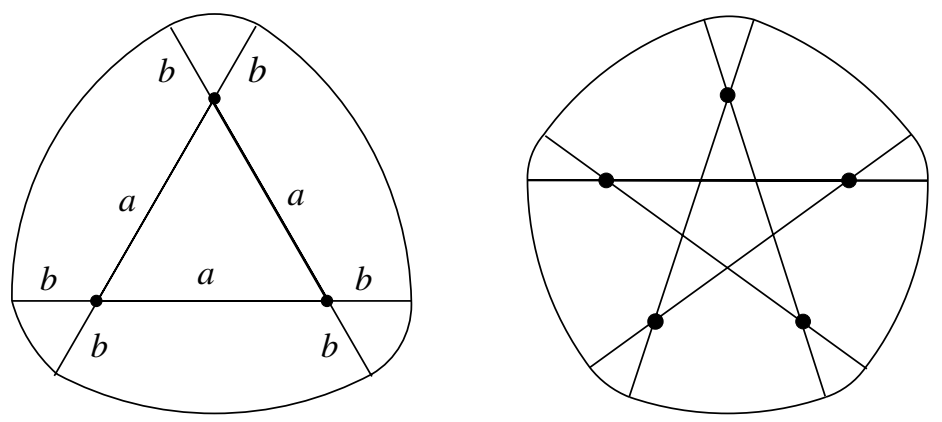

Fig. 1

The theory of curves of constant breadth has generated a considerable literature, starting with Euler [3] and including works by Hurwitz [4], Minkowski [6], Blaschke (five articles in [1]) and many others. These curves have many interesting properties, the most startling being perhaps that their perimeter is $\pi$ times their diameter. See [8, chap. 25] for an elementary account, or [5] for a more thorough, though old, presentation. For $n \geq 3$, to our knowledge the property $C_{n}(D)$ has not been investigated (but see [5, p. 61], and [2] for a related problem).

In this note, we shall prove the theorem below, using only basic differential calculus. We recall that a curve is regular if it is $\mathcal{C}^{1}$ with non vanishing derivative.

\section{Theorem.}

i) For all $n \geq 2$ and $D>0$, there are $\mathcal{C}^{\infty}$ regular simple closed non circular curves $\Gamma$ with property $C_{n}(D)$.

ii) The circle of radius $D / 2$ is the only $\mathcal{C}^{2}$ regular simple closed curve which satisfies both $C_{4}\left(\frac{D}{\sqrt{2}}\right)$ and $C(D)$.

Remarks. For i) $n=2$, such $\mathcal{C}^{\infty}$ curves abound in the literature, see for instance [10] or [7] for one given by a polynomial equation. We however give a short self contained proof that yields simple explicit examples. Notice that ii) gives a definition of the circle involving only Euclidean distance between points on the circle, while the usual definition refers to a point off the circle.

Proof of i) for $n=2$. (Inspired by Euler [3] and following G. Wanner's comments.) To simplify we assume that $D=2$. We start with a stick of length 2 which we place horizontally with center 0 at the origin. We then attach a needle at a point at position $-r$ from 0 and we perform a small rotation of angle $d \phi$ around this point. Then at any moment we change the position $r(\phi)$ of the needle and rotate the stick. As seen in Fig. 2, the center of the stick then describes a curve $(x(\phi), y(\phi))$ with

$$
d x=-r(\phi) \sin \phi d \phi, \quad d y=r(\phi) \cos \phi d \phi,
$$


while the extremities of the stick follow the curve given by

$$
\gamma(\phi)=(x(\phi), y(\phi))+(\cos \phi, \sin \phi) .
$$

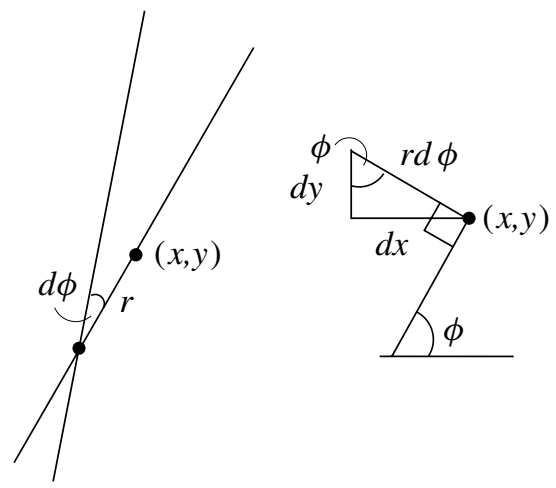

Fig. 2

When the angle $\pi$ is reached, the stick is again horizontal, and the position of the center is at the origin if $x(\pi)=y(\pi)=0$. The distance between $\gamma(\theta)$ and $\gamma(\phi)$ decreases (strictly if $|r(\theta)|<1$ ) as $\phi$ 'goes away' from $\theta+\pi$, as seen in Fig. 3. (The circle of radius 2 and center $\gamma(\theta)$ is dashed.) The curve obtained has thus constant diameter 2 and satisfies $C(2)$ if $-1<r(\phi)<1$, strictly. Note that the proof works also if $r$ is only piecewise continuous. (A curve is piecewise continuous - or $\mathcal{C}^{1}$, regular, etc - if it is continuous - or $\mathcal{C}^{1}$, regular, etc - everywhere, except possibly at a finite number of points.)

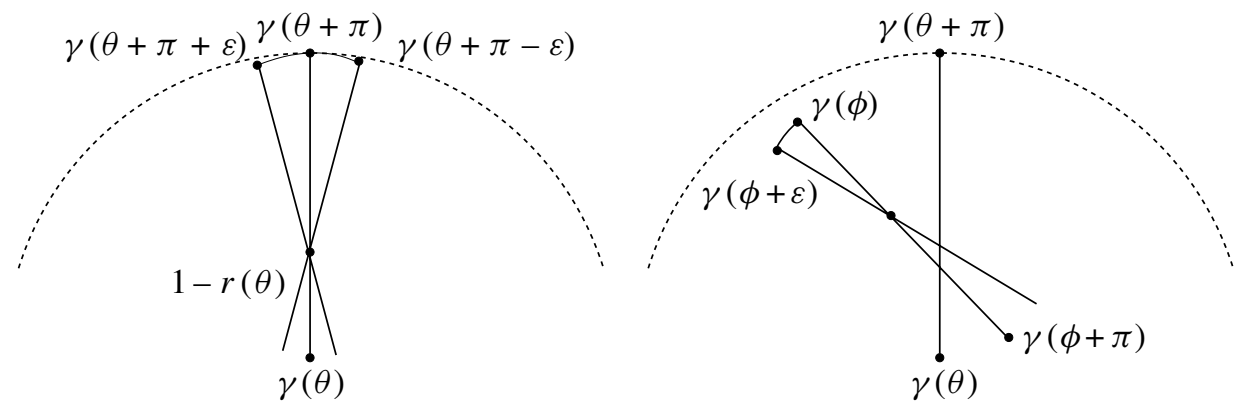

Fig. 3

To summarize, we proved:

Proposition 1. Let $r: \mathbb{R} \rightarrow \mathbb{R}^{2}$ be piecewise continuous and

$$
x(\theta)=-\int_{0}^{\theta} r(\phi) \sin (\phi) d \phi, \quad y(\theta)=\int_{0}^{\theta} r(\phi) \cos (\phi) d \phi .
$$


Suppose that

a) $r(\theta+\pi)=r(\theta),|r(\theta)| \leq \frac{D}{2}$,

b) $(x(0), y(0))=(x(\pi), y(\pi))=(0,0)$.

Then, the curve $\gamma(\theta)=(x(\theta), y(\theta))+\frac{D}{2}(\cos \theta, \sin \theta)$ has constant diameter $D$, and has property $C(D)$ if $|r(\theta)|<\frac{D}{2}$.

Notice that given the path of the center $(x(\phi), y(\phi))$, the coordinate on the stick of the 'point of instant rotation' $r(\phi)$ is given by the equation $x^{\prime}(\phi)=-r(\phi) \sin (\phi)$. The curves in Fig. 1 are obtained by taking $r$ piecewise constant. To obtain $\mathcal{C}^{\infty}$ explicit examples, we can take $r(\theta)=a \cdot \sin ((2 k+1) \theta)$, with $k \geq 1, a<D / 2$. Integrating, we get

$$
(x(\theta), y(\theta))=\frac{a}{4}\left(-\frac{\sin (2 k \theta)}{k}+\frac{\sin (2(k+1) \theta)}{k+1},-\frac{\cos (2 k \theta)}{k}-\frac{\cos (2(k+1) \theta)}{k+1}\right) .
$$

By Proposition 1, the curve $\gamma(\theta)=(x(\theta), y(\theta))+\frac{D}{2}(\cos \theta, \sin \theta)$ has property $C(D)$. One could also take any linear combination of $\cos ((2 k+1) \theta)$ and $\sin ((2 k+1) \theta)(k \geq 1)$, with small enough coefficients, for $r(\theta)$. In Fig. 4 are pictured the curves of diameter 1 (and $(x, y))$ for

$$
r(\theta)=\sin (3 \theta) / 3+\cos (3 \theta) / 5, \sin (5 \theta) / 2.01, \sin (3 \theta) / 10+\cos (7 \theta) / 2.501 .
$$

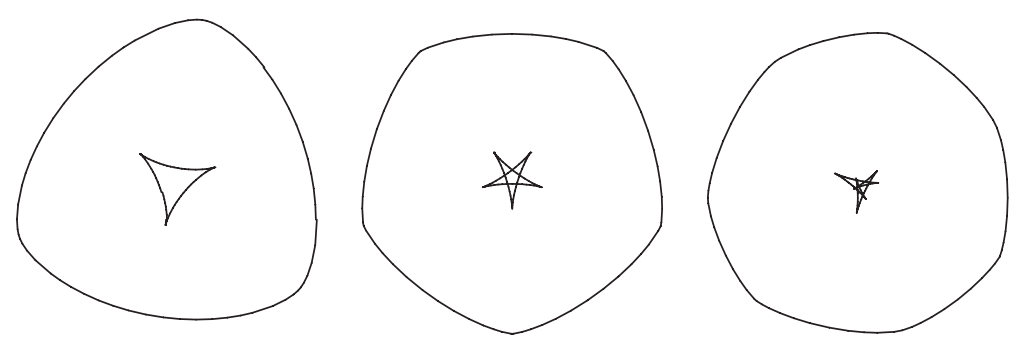

Fig. 4

Proof of i) for $n \geq 3$. We use again G. Wanner's needle idea, but here we have more liberty. Take a regular $n$-gon which we rotate with angle $\phi$ from 0 to $2 \pi / n$. At any moment we have two degrees of freedom to place our needle at a point inside the body of the $n$-gon, with coordinates $(\xi(\phi), \eta(\phi))$ in a coordinate system moving with the $n$ gon. With the notations of Fig. 5 the path $(x(\phi), y(\phi))$ of the center is determined by $d x=-r \sin (\phi+\psi) d \phi, d y=r \cos (\phi+\psi) d \phi$, and after expanding $\sin (\phi+\psi)$ and $\cos (\phi+\psi)$ we get

$$
d x=-(\xi \sin (\phi)+\eta \cos (\phi)) d \phi, \quad d y=(-\eta \sin (\phi)+\xi \cos (\phi)) d \phi .
$$

Then, the condition for the curve described by the vertices of the $n$-gon to be closed is

$$
\int_{0}^{\frac{2 \pi}{n}}(\xi(\phi) \sin (\phi)+\eta(\phi) \cos (\phi)) d \phi=\int_{0}^{\frac{2 \pi}{n}}(-\eta(\phi) \sin (\phi)+\xi(\phi) \cos (\phi)) d \phi=0
$$




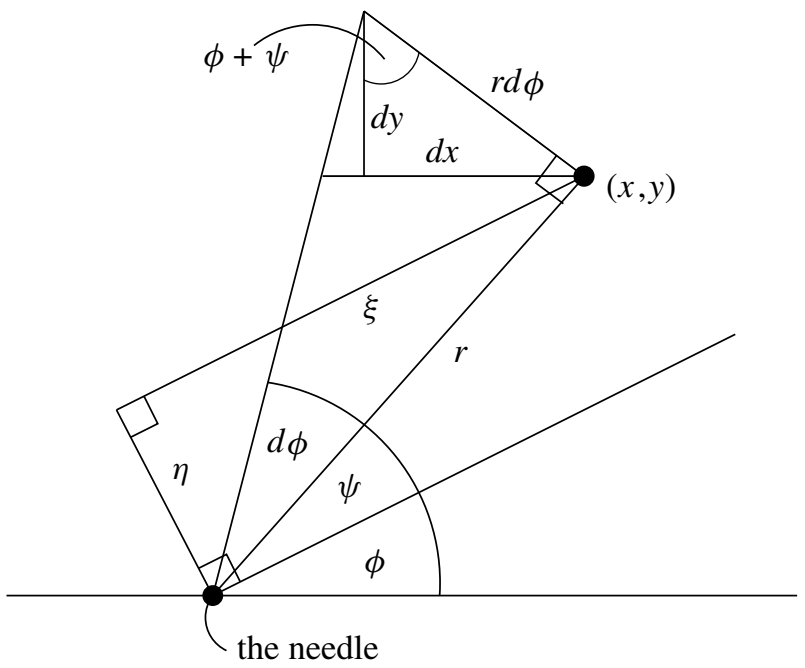

Fig. 5

By construction there is of course an $n$-gon inscribed at each point, and since there are exactly two points at distance $D$ from a given point, this $n$-gon is unique.

To obtain explicit examples it might be easier to start with $x(\phi), y(\phi)$ satisfying $x(\phi+$ $\left.\frac{2 \pi}{n}\right)=x(\phi), y\left(\phi+\frac{2 \pi}{n}\right)=y(\phi)$ and let $\gamma(\phi)=(x(\phi), y(\phi))+R(\cos (\phi), \sin (\phi))$, where $R$ is the radius of the $n$-gon with edges of length $D$. Then if $x, y$ and their derivatives are small enough so that we do not create 'new' points at distance $D$ from a given point, we obtain a curve satifying $C_{n}(D)$.

Proof of ii). Let $\Gamma$ be a $\mathcal{C}^{2}$ regular simple closed curve satisfying $C_{4}(D / \sqrt{2})$ and $C(D)$. We take $D=1$ for simplicity. Let $\gamma$ parametrise $\Gamma$ by arc length counterclockwise.

If $x \in \Gamma$, denote by $c(x)$ the unique point of $\Gamma$ for which $\|x-c(x)\|=1$. If $\gamma(t)=x$, $\gamma^{\prime}(t)$ must be normal to $x-c(x)$, because the curve must be totally inside the "dashed eye" of Fig. 6, and in fact, $c(x)$ is the point on the normal at $x$ at distance 1 from $x$.

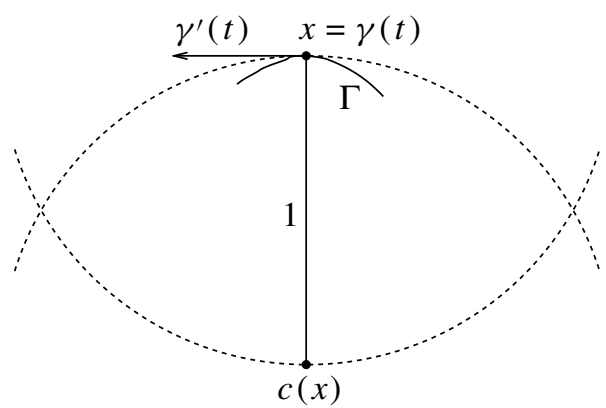

Fig. 6 
Since the unit normal vector at $\gamma(t)$ is $n(t)=\left(-\gamma_{2}^{\prime}(t), \gamma_{1}^{\prime}(t)\right), c(\gamma(t))=\gamma(t)+n(t)$ is differentiable in $t$. (Notice that we cannot use the implicit function theorem here, because the derivative of $\|x-z\|$ vanishes precisely at $z=c(x)$.) Moreover, the angle between the oriented line segment $[\gamma(t), c(\gamma(t))]$ and the horizontal axis strictly increases. Given $\theta \in[0,2 \pi]$, there is thus a unique (oriented) line segment $[x, c(x)]$ (the stick) making an angle $\theta$ with the horizontal axis, we define $G(\theta)=(x(\theta), y(\theta))$ to be (the coordinates of) its middle point. Since $c(x)$ is differentiable with respect to $x, G(\theta)$ is differentiable with respect to $\theta$. Then, $\tilde{\gamma}(\theta)=G(\theta)+\frac{1}{2}(\cos \theta, \sin \theta)$ is a parametrisation of $\Gamma$ (see Fig. 7, on the left). By definition, $c(\tilde{\gamma}(\theta))=\widetilde{\gamma}(\theta+\pi)$, and since the tangent of $\Gamma$ at $x$ is normal to $x-c(x)$, we have

$$
\left\langle\tilde{\gamma}^{\prime}(\theta) \mid(\cos \theta, \sin \theta)\right\rangle=\left\langle G^{\prime}(\theta) \mid(\cos \theta, \sin \theta)\right\rangle=0,
$$

which implies $G^{\prime}(\theta)=r(\theta)(-\sin \theta, \cos \theta)$ for some continuous $r$. (Recall that $r$ gives the coordinate on the stick of the point of instant rotation.)

Now, since $\Gamma$ has property $C_{4}(1 / \sqrt{2})$, there is a unique square $S(\theta)$ with edges of length $\frac{1}{\sqrt{2}}$ inscribed in $\Gamma$ at $\tilde{\gamma}(\theta)$. Since $\tilde{\gamma}(\theta+\pi)$ is the unique point of $\Gamma$ at distance 1 from $\widetilde{\gamma}(\theta), \tilde{\gamma}(\theta+\pi)$ is the vertex of $S(\theta)$ diagonal to $\tilde{\gamma}(\theta)(S(\theta)$ has diagonal 1). Thus, $G(\theta)$ is also the center of $S(\theta)$, which implies $G(\theta+\pi / 2)=G(\theta)$ (see Fig. 7, on the right).
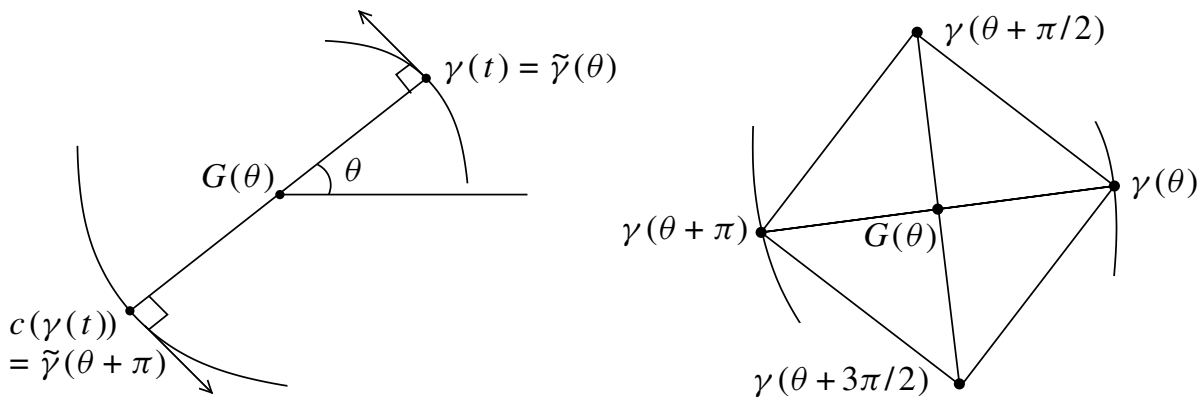

Fig. 7

Therefore, $G^{\prime}(\theta+\pi / 2)=G^{\prime}(\theta)$, i.e. $r(\theta+\pi / 2)(\cos \theta, \sin \theta)=r(\theta)(-\sin \theta, \cos \theta)$, so $r(\theta)=0, G(\theta)$ is constant, and hence $\Gamma$ is a circle.

The end of the proof can also be seen as follows. Since we rotate a square, the needle must stay on the first diagonal of the square on the first half of the trajectory in order to have $C(1)$. But on the second half it must stay on the second diagonal, and thus it must stay in the center and the curve is a circle.

This proof can be easily generalized to show that if a $\mathcal{C}^{2}$ regular closed simple curve has both properties $C_{2 n}(D)$ and $C(R)$, with $R$ two times the radius of the regular $2 n$-gon with edges of length $D$, then it is the circle. This however leaves open the following:

Questions. Are there $D, \widetilde{D}>0$ and curves other than the circle which have property $C(D)$ and $C_{n}(\widetilde{D})$ for some $n \geq 3$ ? 
We finish with the following proposition, which gives a motivation for the "!" in the definition of $C(D)$ :

Proposition 2. Let $\Gamma$ be a continuous closed piecewise regular curve satisfying $C(D)$. Then, $\Gamma$ is regular.

The proof is an exercise for the interested reader. (Hint: Show that $c(x)$ is continuous in $x$, then that $\Gamma$ cannot have corners.) Notice that taking $b=0$ in the first figure of this paper yields a curve of constant diameter with corners (the well known Reuleaux triangle).

Acknowledgements. I wish to thank G. Wanner, who gave so many suggestions for improvements that he should be listed as a co-author, and B. Dudez, librarian at Geneva's Department of Mathematics, for his bibliographical help. I thank also D. Cimasoni.

\section{References}

[1] Blaschke, W.: Gesammelte Werke. Vol. 3. Thales-Verl., Essen 1982-1986.

[2] Eggleston, H.G.; Taylor, S.J.: On the size of equilateral triangles which may be inscribed in curves of constant width. J. London Math. Soc., 27 (1952), 438-448.

[3] Euler, L.: De curvis triangularibus. Acta Acad. Petropol. 1778, II: 3-30, 1780. Reprinted in Opera Omnia, vol. 28.

[4] Hurwitz, A.: Sur quelques applications géométriques des séries de Fourier. Ann. Sci. École Norm. Sup., Sér. 3, 19 (1902), 357-408.

[5] Jordan, Ch.; Fiedler, R.: Contribution à l'étude des courbes convexes fermées. Hermann, Paris 1912.

[6] Minkowski, H.: Über die Körper konstanter Breite. In: Gesammelte Abhandlungen. Vol. II, 277-279. Chelsea Publ., New York 1967. Reprint of the Leipzig Edition, 1911.

[7] Rabinowitz, S.: A polynomial curve of constant width. Missouri J. Math. Sci. 9 (1997) 1, 23-27.

[8] Rademacher, H.; Toeplitz, O.: The enjoyment of mathematics. Princeton University Press, Princeton, N.J., 1957.

[9] Reuleaux, F.: Theoretische Kinematik. Braunschweig 1875. Available at http://historical. library. cornell.edu/kmoddl/toc_reuleauxl.html.

[10] Robertson, S.A.: Smooth curves of constant width and transnormality. Bull. London Math. Soc. 16 (1984) 3, 264-274.

Mathieu Baillif

Université de Genève

Section de mathématiques

C.P. 64

CH-1211 Genève 4, Suisse

e-mail: Mathieu.Baillif@unige.ch 